\title{
The Del1 deposition domain can immobilize 3 $\alpha$-hydroxysteroid dehydrogenase in the extracellular matrix without interfering with enzymatic activity
}

\author{
Chiaki Hidai · Hisataka Kitano · Shinichiro Kokubun
}

Received: 14 June 2008/Accepted: 29 October 2008/Published online: 19 November 2008

(C) The Author(s) 2008. This article is published with open access at Springerlink.com

\begin{abstract}
Developing methods that result in targeting of therapeutic molecules in gene therapies to target tissues has importance, as targeting can increase efficacy and decrease off target-side-effects. Work from my laboratory previously showed that the extracellular matrix protein Del1 is organized in the extracellular matrix (ECM) via the Del1 deposition domain (DDD). In this work, a fusion protein with DDD was made to assay the ability to immobilize an enzyme without disrupting enzymatic function. A prostatic cancer-derived cell line LNCap that grows in an androgendependent manner was used with $3 \alpha$-hydroxysteroid dehydrogenase (3 $\alpha \mathrm{HD}$ ), which catalyzes dihydrotestosterone (DHT). Plasmids encoding a $3 \alpha \mathrm{HD}$ :DDD fusion were generated and transfected into cultured cells. The effects of $3 \alpha \mathrm{HD}$ immobilized in the ECM by the DDD were evaluated by monitoring growth of LNCap cells and DHT concentrations. It was demonstrated that the DDD could immobilize an enzyme in the ECM without interfering with function.
\end{abstract}

Keywords Del1 · Extracellular matrix · Immobilization · Gene therapy

\footnotetext{
C. Hidai $(\bowtie) \cdot$ S. Kokubun

Department of Biomedical Sciences,

Nihon University School of Medicine,

30-1 Oyaguchikami-cho, Itabashi-ku,

Tokyo 173-8610, Japan

e-mail: hidai@med.nihon-u.ac.jp

H. Kitano

Department of Dental Surgery,

Nihon University School of Medicine,

Tokyo 173-8610, Japan
}

\section{Introduction}

Mouse Del1 is an ECM protein secreted by embryonic endothelial cells and hypertrophic chondrocytes [1]. Del1 consists of five domains: three epidermal growth factor (EGF) repeat domains (E1, E2, E3) and two Discoidin domains $(\mathrm{C} 1, \mathrm{C} 2)$. Work from my laboratory recently showed that the $\mathrm{C}$-termini of the $\mathrm{C} 1$ domains are essential for organization of Del1 into the ECM and that both the E3 repeat domain and the $\mathrm{N}$-terminus of the $\mathrm{C} 1$ domain play supportive roles in organization into the ECM [2]. We termed this portion of the $\mathrm{C} 1$ domain the Del1 deposition domain (DDD). Fusion proteins that include the DDD and an alkaline phosphatase protein as a marker accumulate in the ECM without interfering with enzyme activity of the alkaline phosphatase.

In some therapeutic treatments, localization of bioactive molecules specifically to the target tissue(s) increases the concentration of bioactive molecules in the target cells. This results in higher efficiency of treatment and because the concentration of the bioactive molecules in non-target tissues is minimized, this can also result in a lower incidence of treatment side effects. The purpose of this study is to ask if addition of DDD to an enzyme can result in localization of that enzyme to the ECM without affecting activity of the enzyme. Because the space between a cell and the ECM is not a simple vacancy, it is important to ask if a fusion protein derived from an enzyme is functional in the microenvironment of the ECM.

To address this, I designed a cell-based system in which it is possible to readily monitor both an enzyme and the enzymatic products it produces. LNCap cells are prostatic cancer-derived cells that grow in an androgendependent manner and these cells were used as a model target cell in this study [3, 4]. DDD was used to 

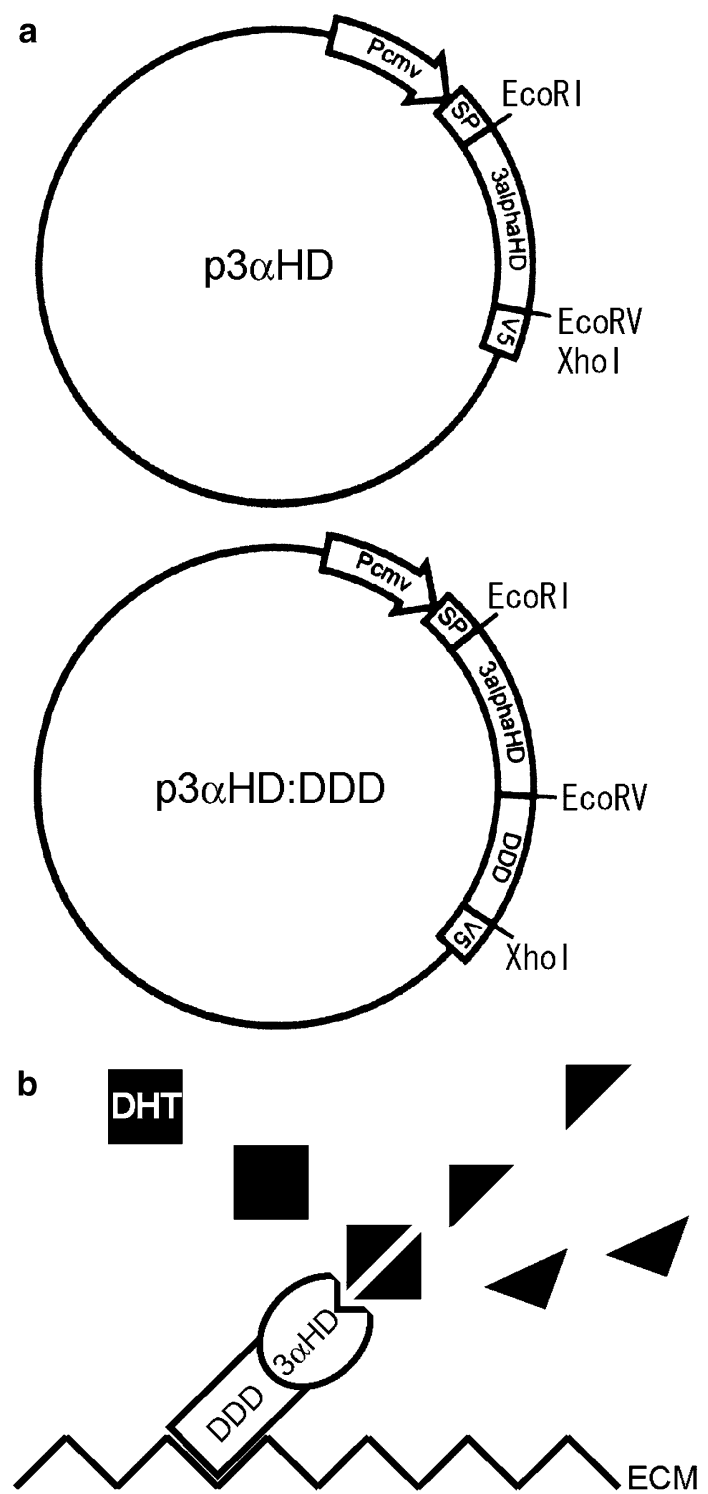

Fig. 1 A schematics for experiments. a DNA constructs generated for transfection experiments. Following CMV promoter sequences (Pcmv), cDNA of signal peptide (SP), cDNA of $3 \alpha \mathrm{HD}$ gene (3alphaHD) and cDNA of DDD (DDD) were inserted into pcDNA3D. b A schematic cartoon of transfection experiments. A recombinant protein of $3 \alpha \mathrm{HD}$ with DDD is represented by an ellipsoid $(3 \alpha \mathrm{HD})$ with a rectangle (DDD). Filled squares and filled triangles indicate DHT and catalyzed DHT, respectively. A zigzag line indicates ECM

immobilize $3 \alpha$-hydroxysteroid dehydrogenase ( $3 \alpha \mathrm{HD})$, which intracellularly catalyzes the conversion of aldehydes and ketones to alcohols, and converts DHT to $5 \alpha-$ androstane-3 $\alpha$-, $17 \beta$-diol physiologically [5]. In this study, recombinant $3 \alpha \mathrm{HD}$ proteins were secreted extracellularly as a result of addition of a signal sequence at the $\mathrm{N}$ terminus of the recombinant protein (Fig. 1a, b). The addition of a DDD to the $3 \alpha \mathrm{HD}$ protein successfully localized the protein to the ECM. Moreover, the $3 \alpha \mathrm{HD}$ in
ECM was biochemically active and suppressed growth of LNCap cells.

\section{Methods}

Cell culture and evaluation of cell growth

LNCap (CRL-1740) and Cos-7 (CRL-1651) cells were purchased from ATCC and grown in $\alpha$-minimum essential medium (Invitrogen, Carlsbad, CA) supplemented with $10 \%$ fetal bovine serum (Invitrogen) at $37^{\circ} \mathrm{C}$ in an atmosphere containing $5 \% \mathrm{CO}_{2}$. To evaluate dependency of cell growth on DHT, 50\% confluent LNCap cells were prepared in a 24 well plate with $300 \mu$ of a serum free medium, VP-SFM (Invitrogen) and different concentrations of DHT (Wako, Osaka, Japan) were added. After 4 days of culture, $30 \mu \mathrm{l}$ of WST-1 (Takara, Outs, Japan) were added to the medium, incubated for 1 hour, and the absorbance at $405 \mathrm{~nm}$ was measured. The experiment was repeated three times and representative data are shown.

\section{DNA constructs}

First, a synthetic oligonucleotide encoding the signal peptide from Del1 (MKHLVAAWLLVGLSLGVPQFGKGDI) was obtained and cloned into pcDNA3D (Invitrogen) resulting in pcDNA3S. Next, a cDNA fragment encoding the human $3 \alpha \mathrm{HD}$ gene (AB178898) was amplified by reverse transcriptase-PCR (RT-PCR) using the forward primer, 5'-AAGAATTCATGAACTCCAAATGTCATTG TGTCAT and the reverse primer 5'-AAGATATCGT ATTCATCCAAAAATGGCCAATTAG. The amplified fragment was cloned into the $3^{\prime}$ end of the signal peptide, resulting in $\mathrm{p} 3 \alpha \mathrm{HD}$ (Fig. 1a). Finally, a fragment encoding the DDD (amino acids 122-316 of Del1) was amplified with the forward primer 5'-AAGATATCTGTGAAG CTGAGCCTTGCAGAAAT and the reverse primer 5'-A ACTCGAGCATGTCCATGTTGAGTGTTCTGAA. This was cloned into the $3^{\prime}$ end of the $3 \alpha \mathrm{HD}$ gene, resulting in the plasmid p $3 \alpha$ HD:DDD (Fig. 1a). The recombinant proteins expressed by these constructs also had a V5 epitope tag at their $\mathrm{C}$ terminal ends.

Immunoblotting

ECM samples for immunoblotting analysis were prepared according to Hidai et al. [2]. Briefly, Cos-7 cells in six-well plate were transfected with pcDNA3S, p3 $\alpha \mathrm{HD}$ or p $3 \alpha$ HD:DDD using Lipofectamine 2000 (Invitrogen). After $96 \mathrm{~h}$ of culture, conditioned medium was harvested. Then, cells were induced to detach by treatment of EDTA 
and the remaining ECM was collected with a scraper. The conditioned medium, cells, and ECM were fixed with $10 \%$ trichloracetic acid (Wako). The protein samples were separated by SDS-PAGE and transferred to a PVDF membrane (ATTO, Tokyo, Japan). The membrane was incubated with anti-V5 antibody (Invitrogen), followed by incubation with a horseradish peroxidase-conjugated secondary antibody, and immunoreactive proteins were detected using the ECL Advance Western Blotting Detection Kit (Amersham, Piscataway, NJ).

\section{Evaluation of the effects of exogenous $3 \alpha \mathrm{HD}$}

LNCap cells were plated to $50 \%$ confluency in a 24 -well dish and transfected with $1 \mu \mathrm{g}$ of pcDNA3S, p3 $\alpha \mathrm{HD}$, or p3 $\alpha$ HD:DDD using Lipofectamine2000. To test if an excess of $\mathrm{p} 3 \alpha \mathrm{HD}$ was introduced, cell growth was monitored after introduction of various amount of $\mathrm{p} 3 \alpha \mathrm{HD}$. To monitor transfection efficiency, $0.1 \mu \mathrm{g}$ of cDNA for the LacZ gene was co-transfected with each test construct. Expression of $L a c Z$ gene was monitored by measuring $\beta$ galactosidase enzyme activity using an assay system (Promega, Madison, WI). Cell number was evaluated with WST-1. The values obtained in samples transected with the control plasmid, pcDNA3S, were normalized to 1 . The experiment was repeated three times and representative data are shown.

\section{Assays of conditioned ECM}

ECM conditioned with $3 \alpha \mathrm{HD}$ was prepared according to Hidai et al. [2]. Briefly, Cos-7 cells were cultured in a 24well dish and transfected with $1 \mu \mathrm{g}$ of pcDNA3S, p3 $\alpha \mathrm{HD}$, or p3 $\alpha$ HD:DDD using Lipofectamine2000. To standardize transfection efficiency, $0.1 \mu \mathrm{g}$ of LacZ cDNA were cotransfected. Four days later, the cells were washed with phosphate buffered saline (PBS) and incubated in PBS containing $10 \mathrm{mM}$ EDTA and a protease inhibitor cocktail (PIERCE, Rockford, IL) for $12 \mathrm{~h}$ at $4^{\circ} \mathrm{C}$. Next, cells were harvested by vigorous pipetting, collected by centrifugation, resuspended in $100 \mu \mathrm{l}$ of tris buffered saline (TBS), and lysed by sonication. $\beta$-Galactosidase activity of the cell lysates was then assayed. Next, $1 \times 10^{4}$ LNCap cells were plated on the conditioned ECM, cultured for seven days, and evaluated with WST-1. To ask if exogenous DHT could rescue the growth of LNCap cells, various amounts of DHT (Wako) were added to the medium. The values obtained for samples transfected with the control plasmid, pcDNA3S, were normalized to 1 . The experiment was repeated three times and representative data are shown.
Measurement of dihydrotestosterone levels

ECM conditioned with $3 \alpha \mathrm{HD}$ was prepared as described above. Serum obtained from a male volunteer as approved by Nihon University ethics committee was incubated with conditioned ECM in the presence of a protease inhibitor cocktail (PIERCE) for $96 \mathrm{~h}$. The concentration of dihydrotestosterone in the serum was measured using the Dihydrotestosterone ELISA kit (IBL, Hamburg, Germany) according to the manufacture's protocol. The values obtained for samples transfected with the control plasmid, pcDNA3S, were normalized to 1 . The experiment was repeated three times and representative data are shown.

Statistical analysis

Results were expressed as mean \pm SEM. Dunn's tests or a Wilcoxon's test were performed, and statistical significance was set at $P<0.01$.

\section{Results and discussion}

To confirm characteristics of LNCap cells, the cells were cultured in serum-free medium with various concentrations of DHT. As previously reported [3, 4], growth of LNCap cells was dose-dependently accelerated by addition of exogenous DHT (Fig. 2a). Next, the distribution of recombinant proteins in cells, ECM and medium was examined. Cells transfected with the control plasmid pcDNA3S, which encodes only a signal peptide (too small to be detected by SDS-PAGE as shown in Fig. 2b), were used as a negative control. The expected sizes of the recombinant proteins encoded by $\mathrm{p} 3 \alpha \mathrm{HD}$ and $\mathrm{p} 3 \alpha \mathrm{HD}$ :DDD were $43 \mathrm{kDa}$ and $65 \mathrm{kDa}$, respectively (Fig. 2b). The recombinant protein encoded by $\mathrm{p} 3 \alpha \mathrm{HD}$ was not detected in ECM samples but was detected in conditioned medium and in cells. The recombinant protein encoded by $\mathrm{p} 3 \alpha \mathrm{HD}$ :DDD was localized to the ECM as expected for a DDD-containing polypeptide. The results also show that the total amount of $3 \alpha \mathrm{HD}$ :DDD present in the culture medium was greater than the total amount found in the ECM. However, considering that the volume of medium was much larger than the volume of ECM tested, the concentration of $3 \alpha \mathrm{HD}$ :DDD was much higher in ECM than in medium. Thus, it appears that the recombinant protein was concentrated in the ECM by the presence of the DDD.

To ask if recombinant proteins with $3 \alpha \mathrm{HD}$ affect growth of LNCap cells, plasmids encoding various $3 \alpha \mathrm{HD}$-derived constructs were transfected into LNCap cells. Transfection with $\mathrm{p} 3 \alpha \mathrm{HD}$ or $\mathrm{p} 3 \alpha \mathrm{HD}$ :DDD resulted in reduction of cell growth at day 6 (Fig. 3a). To check that transfection efficiency did not vary grossly, transfection efficiency was 

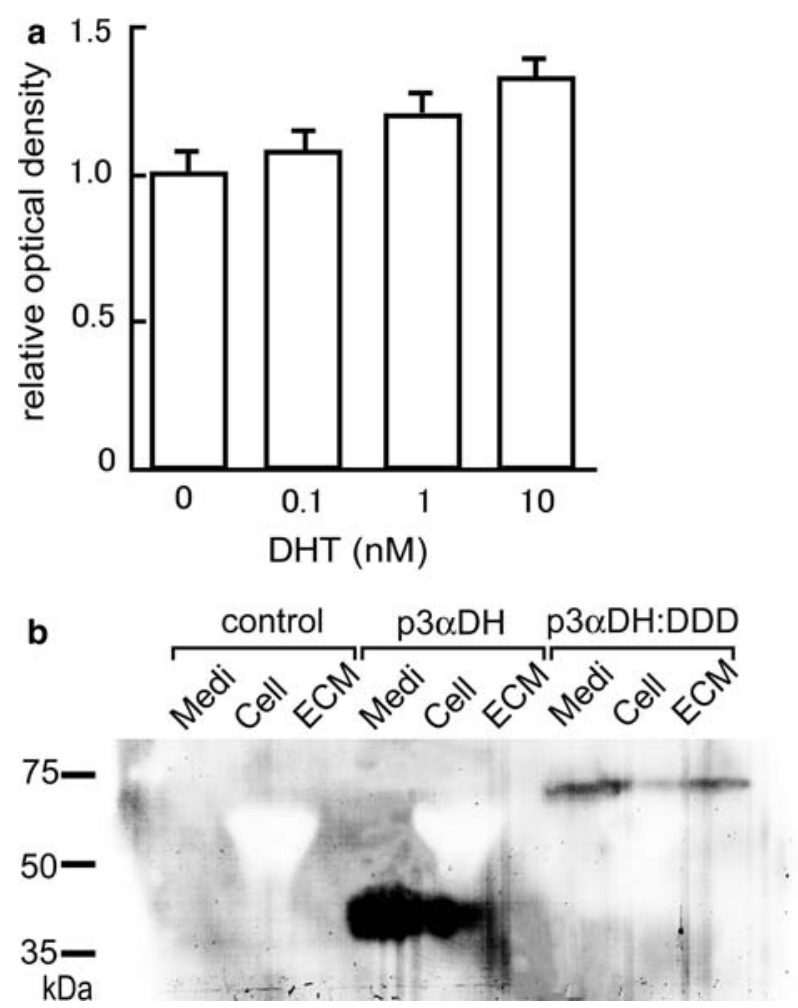

Fig. 2 A cell-based readout of enzymatic activity. a Growth dependence of LNCap cells on DHT. The results represent mean \pm SEM $(n=6)$. b Immunoblotting analysis reveals expression of recombinant proteins in cultured medium (Medi), cells, and ECM

monitored by assaying $\beta$-galactosidase activity after cotransfection with a LacZ construct. Transfection of p3 $\alpha$ HD:DDD seemed to be more effective than that of p3 $\alpha \mathrm{HD}$ (Fig. 3a). In this study, it was unfortunately impossible to standardize protein levels. Strictly speaking, our results cannot address if DDD improves the efficiency or effects of an enzyme by concentrating it in the ECM, as would be relevant to therapeutics [6]. The question may be resolved in an in vivo study, wherein not only the distribution but also the effects and side effects of the recombinant proteins could be evaluated.

To determine if there is a dose-dependent relationship between cell growth and the amount of $\mathrm{p} 3 \alpha \mathrm{HD}$ DNA transfected into cells, the effect of transfection with different amounts of p $3 \alpha \mathrm{HD}$ DNA was tested (Fig. 3b). The effect on cell growth was dependent on the amount of p $3 \alpha \mathrm{HD}$ added, suggesting that suppression of cell growth was not due to experimental procedures but instead, is the result of $3 \alpha \mathrm{HD}$-specific effects.

DHT may be catabolized in cells transfected with the $3 \alpha \mathrm{HD}$ construct; thus, I next assayed if a growth suppression effect is induced by ECM conditioned with p $3 \alpha$ HD:DDD (Fig. 4a). To again check that the transfection efficiency did not vary grossly, transfection efficiency was monitored by assaying $\beta$-galactosidase activity.

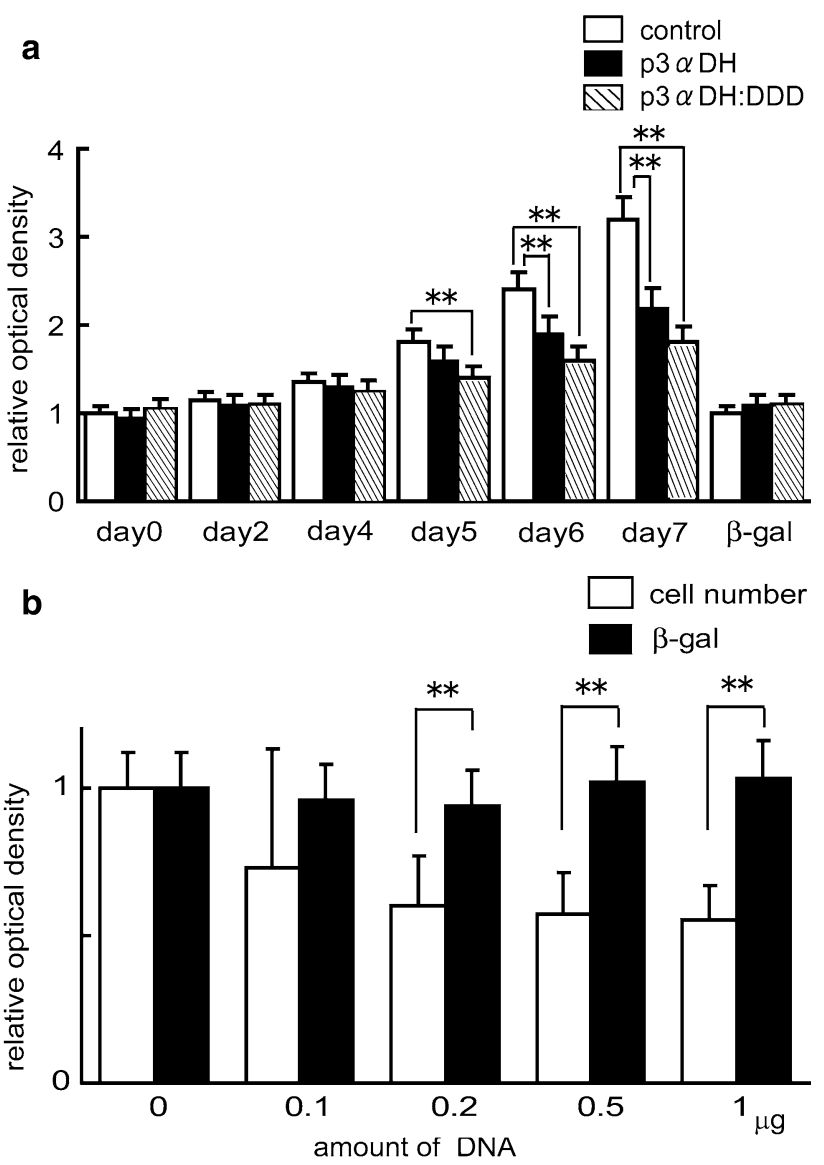

Fig. 3 Exogenous expression of $3 \alpha \mathrm{HD}$ in LNCap cells affects cell growth. a Growth of LNCap cells was evaluated after transfection with the plasmids $\mathrm{p} 3 \alpha \mathrm{HD}$ or $\mathrm{p} 3 \alpha \mathrm{HD}$ :DDD. A gene encoding $\beta$ galactosidase ( $\beta$-gal) was co-transfected as a control and $\beta$-gal activity was used as a measure of transfection efficiency. $\mathbf{b}$ Growth of LNCap cells was evaluated after introduction of different amounts of the $\mathrm{p} 3 \alpha \mathrm{HD}$ construct. Open bars LNCap cell numbers; closed bars $\beta$ gal activity as a measure of transfection efficiency. The results represent mean $\pm \operatorname{SEM}(n=6)$. $* * P<0.01$

Growth of cells on ECM conditioned by cells transfected with $\mathrm{p} 3 \alpha \mathrm{HD}$ was not different from growth on control ECM. However, ECM conditioned by cells transfected with $\mathrm{p} 3 \alpha \mathrm{HD}$ :DDD significantly suppressed the growth of otherwise untreated LNCap cells.

On explanation for the effect observed in this work is that the presence of $3 \alpha \mathrm{HD}$ in the ECM decreases the concentration of DHT in serum. To test this, serum was incubated with conditioned ECM and the concentration of DHT was measured in treated and untreated samples (Fig. 4b). After $96 \mathrm{~h}$, the concentration of DHT in serum incubated with ECM conditioned with p3 $\alpha$ HD:DDD had significantly decreased.

To confirm that the $\mathrm{p} 3 \alpha \mathrm{HD}$ :DDD suppresses the growth of LNCap cells via a decrease of DHT in medium, the ability of exogenously added DHT to reverse the effect was assayed (Fig. 4c). Administration of exogenous DHT was 

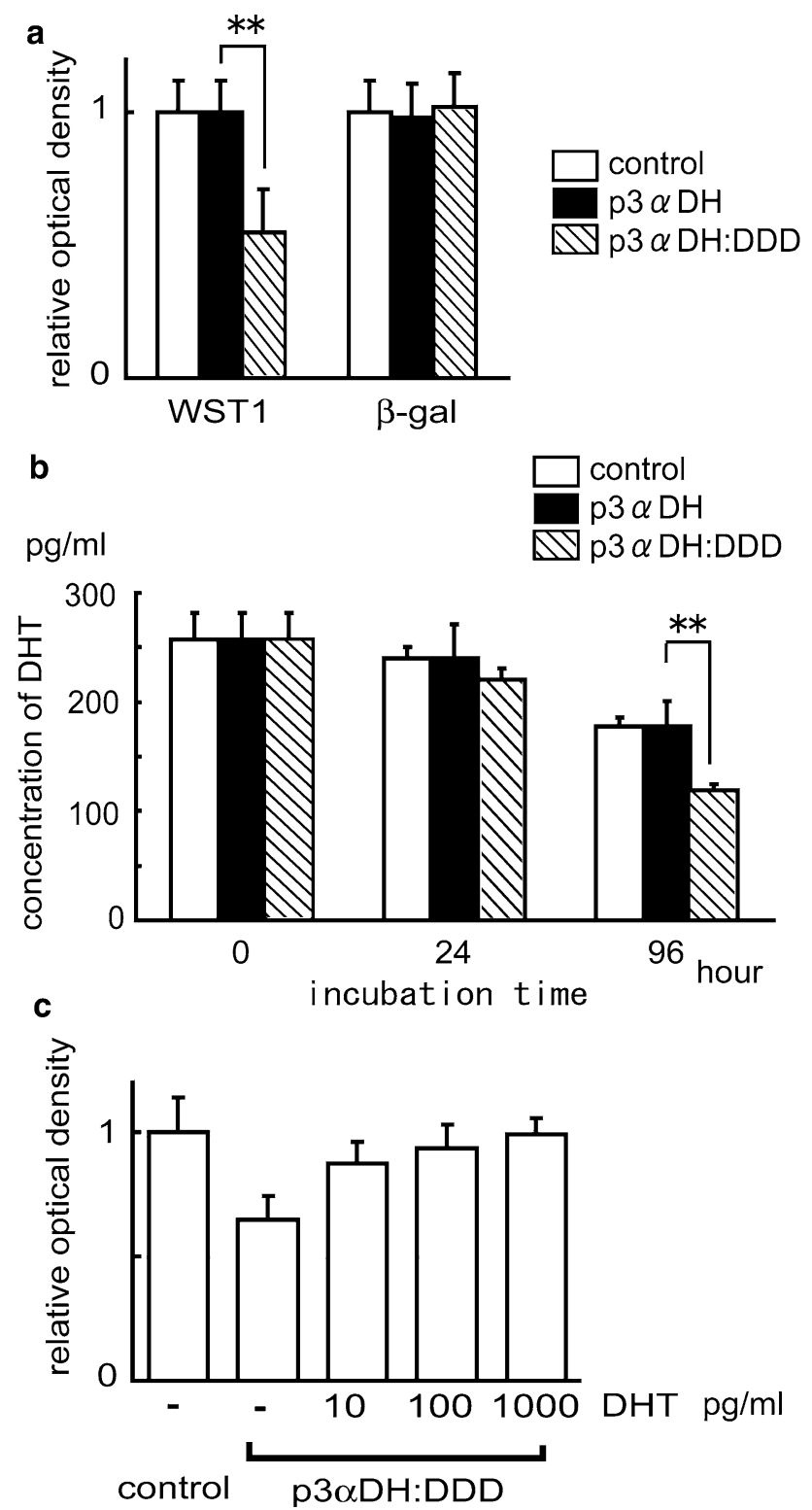

Fig. 4 The effects of $3 \alpha \mathrm{HD}$ localized to ECM by DDD. a The ECM conditioned with p3 $\alpha \mathrm{HD}$ :DDD suppressed the growth of LNCap cells. $\mathrm{LacZ}$ was co-transfected and $\beta$-gal activity was assayed as a measure of transfection efficiency. b Concentration of DHT in serum incubated with $\mathrm{p} 3 \alpha \mathrm{HD}$ :DDD conditioned ECM as measured by ELISA. c Addition of exogenous DHT restored LNCap cell growth normally suppressed by ECM conditioned with p $3 \alpha$ HD:DDD. The results represent mean $\pm \operatorname{SEM}(n=6)$. ${ }^{* *} P<0.01$

able to restore growth of LNCap cells even in the presence of $\mathrm{p} 3 \alpha \mathrm{HD}: \mathrm{DDD}$.

In a previous study, it was found that alkaline phosphatase protein was efficiently deposited in the ECM in vitro and in vivo [2]. The present study suggests that DDD can fix enzymes in the ECM in a functional state, suggesting some biomedical applications for DDD fusion proteins. In cytotherapy, for example, cells could be preengineered with a cDNA encoding a specific DDD fusion protein. Because DDD has been reported to be effective for targeting to ECMs from various kinds of cells, one can predict that cell choice may be dictated solely according to need and that the ECM conditioned by the cells can then serve as a source of active enzyme.

Similarly, a system involving a DDD fusion protein under the control of a tissue-specific promoter could efficiently localize an exogenous protein useful in gene therapy and thus presumably, target the effects of that enzyme to a specific cell type and/or location. This has promise for generating regions of high local concentration while at the same time, resulting in only a low systemic concentration of the therapeutic proteins by virtue of their deposition in ECM. To apply the idea further, one can imagine that chemicals capable of being activated by an enzyme may then by activated in a limited region, such as in a specific target tissue or cell type, via expression of an ECM-anchored version of the activating enzyme.

In addition to enzymes, some ligands, such as the FAS ligand, might be used as partners of DDD and the system adapted to other applications [7]. That is, the presence of a cytotoxic ligand may limit the number of cells that can produce the ligand but if that ligand is in the ECM via anchoring by DDD, the ligand may remain active and accessible in the ECM even after death of the cell that initially expressed it. If this type of fusion between DDD and cytotoxic ligands results in a protein that is fixed in the ECM and significantly active, then one can imagine development of additional applications for the system.

Open Access This article is distributed under the terms of the Creative Commons Attribution Noncommercial License which permits any noncommercial use, distribution, and reproduction in any medium, provided the original author(s) and source are credited.

\section{References}

1. Hidai C, Zupancic T, Penta K et al (1998) Cloning and characterization of developmental endothelial locus-1: an embryonic endothelial cell protein that binds the $\alpha \mathrm{v} \beta 3$ integrin receptor. Genes Dev 12:21

2. Hidai C, Kawana M, Kitano H et al (2007) Discoidin domain of Del1 protein contributes to its deposition in the extracellular matrix. Cell Tissue Res 330:83

3. Horoszewicz JS, Leong SS, Kawinski E et al (1983) LNCaP model of human prostatic carcinoma. Cancer Res 43:1809

4. van Bokhoven A, Varella-Garcia M, Korch C et al (2003) Molecular characterization of human prostate carcinoma cell lines. Prostate 57:205

5. Penning TM, Burczynski ME, Jez JM et al (2000) Human $3 \alpha$-hydroxysteroid dehydrogenase isoforms (AKR1C1-AKR1C4) of the aldo-keto reductase superfamily: functional plasticity and tissue distribution reveals roles in the inactivation and formation of male and female sex hormones. Biochem J 351:67

6. Sawyers C (2004) Targeted cancer therapy. Nature 432:294

7. ElOjeimy S, McKillop JC, El-Zawahry AM et al (2006) FasL gene therapy: a new therapeutic modality for head and neck cancer. Cancer Gene Ther 13:739 